patients would need to be treated to keep one free from seizures in the acute phase. Seizure control was not accompanied by a reduction in mortality (pooled $\mathrm{RR}=1.49$ ) or neurological disability. The occurrence of late seizures was not reduced by AEDs; the relative risk of late seizures, based on 4 studies, was 1.28 . The risk of skin rashes was increased $(R R=1.57)$. The true net benefit of prophylactic antiepileptic agents was undetermined. (Schierhout G, Roberts I. Prophylactic antiepileptic agents after head injury: a systematic review. I Neurol Neurosurg Psychiatry Jan 1998;64:108-112). (Respond: Dr GH Schierhout, Department of Epidemiology and Public Health, Institute of Child Health, University College, 30 Guilford St, London WC1N $1 \mathrm{EH}, \mathrm{UK})$.

COMMENT. Prophylactic antiepileptic drug therapy initiated after acute head injury may reduce the occurrence of seizures in the first week, but has no effect on the development of late seizures, on mortality or neurological disability, and treatment is associated with the risk of skin rash, a potentially serious side effect. For 100 patients treated 10 may be seizure-free in the first week, but 4 will develop skin rash.

See Progress in Pediatric Neurology II, (PNB Publishers, 1994;pp137-138) for reports of prophylactic anticonvulsant drugs after craniotomy. In one study from Walton Hospital, Liverpool, UK, skin rashes occurred in $13 \%$ of patients treated with carbamazepine or phenytoin (Foy PM et al, 1992), and the occurrence of seizures within the first post-operative week did not increase the likelihood of late epilepsy. The authors concluded that drug therapy should not be recommended routinely following craniotomy.

Endocrine functions following severe head trauma were assessed in 21 children at Chaim Sheba Medical Center, Tel-Hashomer, Israel (Goldman M et al. Pediatr Neurol Nov 1997;17:339-343). Advanced bone age without other signs of precocious puberty were found in 3 prepubescent children. Biochemical and hormonal determinations were normal, and no endocrine abnormalities were found in children examined 4 months or more following injury. Clinical monitoring is sufficient, and specific hormonal measurements are required only when warranted by abnormal signs.

\title{
HEADACHE
}

\section{FOOD-RELATED HEADACHES}

The literature relating to food and headache is reviewed by an authority on migraine, Dr Clifford Rose of the London Neurological Centre, London, UK. Wine was the earliest reference to dietary migraine, a comment attributed to Celsus (25BC-50AD). Fothergill (1712-1780) was the first to incriminate chocolate as a precipitant of migraine. More recent studies have postulated the phenylethylamine content as the active provocative ingredient. Martelletti and colleagues (1994) studied the involvement of the immune system, and found a link between interleukins and other cytokines, immune messengers, with histamine and serotonin, neuromediators of pain in migraine. Hanington (1967) proposed the theory of tyramine contained in cheese as a precipitant of dietary migraine, but subsequent studies provided conflicting results. Caffeine withdrawal is one explanation for weekend migraine sufferers. Octopamine, a biogenic amine, is thought to be the active ingredient in citrus fruit headache. Food additives blamed for exacerbations of migraine in some patients include aspartame (diet-soda headache), nitrites and nitrates (hot dog headache), and glutamate (chinese 
restaurant MSG headache). Red wine contains more phenolic flavonoids than white wine, which might explain the reported red wine sensitivity of some migraineurs; sensitivity may be related to a low level of phenosulphotransferase $\mathrm{P}$, an enzyme that detoxicates the flavonoid phenols. Alcohol-induced headache has been related to an increase in body water. Ice cream headache is an accepted phenomenon, but the mechanism is controversial. Fasting is a generally accepted migraine trigger, but hypoglycemia may not be the mechanism. Elimination diets have had mixed results in controlling migraine. The author concludes that apart from a sensitivity to red wine and other alcoholic drinks, proposed dietary triggers require additional factors to induce migraine attacks. (Rose FC. Food and Headache. Headache Q 1997;8:319-329). (Reprints: F Clifford Rose MD, London Neurological Centre, 110 Harley Street, London W1N 1AF, UK).

COMMENT. Diet and headache is a controversial topic that needs further scientific study. Anecdotal evidence and limited controlled experiments are very persuasive for items such as chocolate, milk, cheese, coffee, aspartame, nitrites, MSG, and red wine. However, results of elimination diets and challenge experiments are sometimes conflicting in groups of patients, and individual differences in sensitivity or combinations of factors may explain the controversies. After working with Dr John Wilson in his clinic at Great Ormond Street Hospital, London, I am convinced of a relation between dietary factors and headache in children. (For reviews of articles on Diet and Migraine, see Millichap JG, ed. Progress in Pediatric Neurology Vol I, 1991;pp146-150; Vol II, 1994;pp166168; Vol III, 1997;pp170-171). My experience in the United States, however, is hampered by a reluctance of parents to embark on a time-consuming, hypoallergenic dietary therapy, often requiring the added expense of nutritional counselling and supervision. At best, parents and patients can sometimes be persuaded to try the avoidance of caffeine and aspartame-containing diet sodas, often consumed in extraordinary quantities by young children, and the restriction of chocolate may also be attempted. A suggestion that milk and other dairy products may be the offending headache triggers is frequently received with a look of disbelief. Patients have been educated in reliance on the "pill."

\section{ATENTION DEFICIT AND COMORBID DISORDERS}

\section{METHYLPHENIDATE-INDUCED OBSESSIVE-COMPULSIVENESS}

An 8-year-old boy with a transient but severely debilitating obsessivecompulsive disorder (OCD), induced by a brief course of methylphenidate (MPH) (10 mg/daily) for treatment of mild, uncomplicated attention deficit hyperactivity disorder, is reported from the University of Illinois College of Medicine, Rockford, Illinois. After 2 weeks of MPH treatment, school work improved, but OC behaviors emerged: avoidance of touching objects, repeated hand washing. He also developed tics involving head and neck. The past history was negative for psychiatric comorbidity, recent streptococcal infection, and familial anxiety disorders. Withdrawal of MPH was followed by gradual recovery from the OCD over a 3 month period. (Kouris S. Methylphenidate-induced obsessive-compulsiveness. LAm Acad Child Adolesc Psychiatry Feb 1998;37:135). (Respond: Dr Steven Kouris, University of Illinois College of Medicine, Rockford, IL).

COMMENT. Obsessive compulsive disorder (OCD) is a rarely reported complication of psychostimulant therapy for ADHD. The author cites only two previous references. Dextroamphetamine has been implicated more often than methylphenidate (MPH). Tic disorder is a comorbid complication and a more 